\section{$\underset{\substack{\text { hommes } \\ \text { \& migrations }}}{ }$}

\section{Hommes \& migrations}

Revue française de référence sur les dynamiques

migratoires

\section{$1296 \mid 2012$}

Le Mexique dans les migrations internationales

\title{
Contrôle des frontières
}

Questions de droits humains et d'éthique sur une stratégie états-unienne

\section{Nestor Rodriguez}

Traducteur : Françoise Bouillot

\section{(2) OpenEdition \\ Journals}

\section{Édition électronique}

URL : http://journals.openedition.org/hommesmigrations/1506

DOI : 10.4000/hommesmigrations. 1506

ISSN : 2262-3353

\section{Éditeur}

Musée national de l'histoire de l'immigration

Édition imprimée

Date de publication : 1 mars 2012

Pagination : $54-63$

ISSN : 1142-852X

Référence électronique

Nestor Rodriguez, "Contrôle des frontières », Hommes \& migrations [En ligne], 1296 | 2012, mis en

ligne le 31 décembre 2014, consulté le 19 avril 2019. URL : http://journals.openedition.org/

hommesmigrations/1506 ; DOI : 10.4000/hommesmigrations.1506 


\section{Contrôle des frontières \\ Questions de droils humains et d'éthique sur une stratégie états-unienne}

Par Nestor Rodriguez, professeur de sociologie, université du Texas (Austin, États-Unis)*

Le renforcement de la frontière entre les États-Unis et le Mexique a un effet direct sur ceux qui tentent de la franchir. Face un passage rendu de plus en plus difficile, les migrants n'ont

d'autre choix que de traverser des zones dangereuses.

Pour contourner cette politique de dissuasion, ils risquent leur vie. La police des frontières américaine est en première ligne de ce dispositif. Derrière, c'est toute une bureaucratie qui semble rester sourdle aux conséquences tragiques de sa politique migratoire. 
En 1994, la police des frontières des États-Unis appliqua la stratégie intitulée "Prevention through Deterrence" (prévention par la dissuasion) à la frontière avec le Mexique en fermant plusieurs points de passage très fréquentés par les immigrants clandestins, redirigeant ceux-ci vers des zones de passage inhospitalières et dangereuses. Peu après, le nombre de migrants ayant trouvé la mort en tentant de franchir la frontière s'est accru. Les États-nations ferment leurs frontières en application de leur souveraineté, mais en général à l'aide de moyens raisonnables qui ne causent pas de dommages importants aux migrants, surtout ceux qui franchissent la frontière en quête de travail.

Je m'intéresse ici aux aspects humanistes de cette stratégie de "prévention par la dissuasion" pour le contrôle des frontières, en ciblant notamment quatre questions : pourquoi les responsables de la bureaucratie des frontières, c'est-à-dire de l'Immigration and Naturalization Service (INS) n'ont-ils pas anticipé cette augmentation des décès après l'application de la stratégie de dissuasion ? À quels présupposés et quelles informations sur le comportement des migrants la police des frontières a-t-elle fait appel pour mettre en ceuvre cette stratégie ? Quelle influence les environnements sociaux nationaux et frontaliers ont-ils eu sur son application? Quel degré de causalité et de responsabilité peut-on attribuer à la bureaucratie pour la mort de ces migrants ? L'approche analytique de cet article emprunte aux travaux de Gideon Sjoberg sur les questions éthiques et humaines qui se posent aux bureaucraties ${ }^{(1)}$. Il leur reprend notamment deux présupposés : les bureaucraties sont plus vastes que la somme des individus qui y travaillent, et elles ne sont pas réductibles à ceux-ci. En outre, ces corps constitués sont porteurs d'une responsabilité morale ${ }^{(2)}$.

\section{La prévention par la dissuasion}

Quand la police des frontières annonça en 1994 un renforcement des lois étatsuniennes sur l'immigration, elle présenta un plan donnant la priorité au contrôle de l'immigration clandestine à la frontière entre les États-Unis et le Mexique ${ }^{(3)}$. L'INS approuva à l'été 1994 cette stratégie de prévention par la dissuasion. Il s'agissait de faire cesser (en partie) l'immigration clandestine en "décourageant" les candidats au passage. La première phase de cette stratégie consistait en un renforcement des clôtures et une augmentation des effectifs de police dans les zones frontalières de San Diego et El Paso. La seconde phase ciblait les secteurs de Tucson et du sud du Texas, et la troisième visait le reste de la frontière avec le Mexique ${ }^{(4)}$.

Alors que la première phase de la dissuasion touchait à sa fin en 1997, le nombre de migrants clandestins appréhendés chutait brutalement dans les secteurs de San Diego 
et El Paso, et s'élevait de façon spectaculaire dans les zones sur lesquelles se rabattaient les migrants pour entrer aux États-Unis sans visa. Si l'on prend 1993 pour année de référence, on constate en 1997 que les arrestations dans les secteurs de San Diego et El Paso ont chuté respectivement de $47 \%$ et $56 \%$, mais ont augmenté de plus de $100 \%$ dans quatre des sept autres secteurs du SudOuest $^{(5)}$. Dans les trois secteurs respectifs de Tucson, McAllen et El Centro, qui recèlent de vastes étendues de terrain accidenté et dangereux, les arrestations augmentèrent de plus de 100000 entre 1993 et 1997. À mesure qu'une plus grande proportion de l'immigration clandestine se tournait vers les zones montagneuses, les déserts et la traversée de rivières dangereuses, les chiffres de décès associés au franchissement illégal de la frontière enregistraient trois types de modifications. D'abord, le nombre de décès déclarés de migrants remonta en 1995 après six années de déclin ${ }^{(6)}$.Selon l'étude d'Eschbach et al., du début de la nouvelle politique frontalière en 1994, à la fin de sa première phase en 1997, le nombre de décès était passé de 192 à $224^{(7)}$.

Ensuite, dans le secteur frontalier de Tucson, le nombre de morts fit un bond, excédant désormais 100 pour $2000^{(8)}$. En 2000, le secteur de Tucson comptait le tiers de tous les décès de clandestins ayant tenté de passer la frontière. Enfin, les décès dus aux conditions environnementales (excès de froid ou de chaleur), jusque-là l'une des plus faibles catégories de morts de migrants, devinrent le premier type de mortalité à la fin des années quatre-vingt-dix ${ }^{(9)}$, les migrants ayant succombé dans les zones dangereuses qui restaient les seules options pour entrer sans contrôle. En 2001, l'exposition à l'environnement comptait pour un tiers des décès connus de migrants dans la zone frontalière ${ }^{(10)}$.

Ces modèles de décès de migrants persistèrent dans les années suivantes. En 2005, leur nombre total atteignait 472, et les neuf premiers mois de 2006 montraient un chiffre plus élevé que sur la même période en $2005^{(11)}$. Le secteur de Tucson resta celui qui affichait le plus grand nombre de ces décès connus (216) et la plus grande proportion de décès dus à l'environnement, avec quelques fluctuations selon les années ${ }^{(12)}$. La stratégie de prévention par la dissuasion a introduit une nouvelle routine que critiquent certains agents de la police des frontières. Avant, en effet, ils patrouillaient les territoires frontaliers en cherchant les migrants clandestins ; selon la nouvelle procédure, ils se postaient dans un endroit proche de la frontière où leurs véhicules 
restaient garés pendant des heures bien en vue des migrants, pour décourager ainsi les tentatives de franchissement de la frontière. Ils appelaient cette routine "faire du sur place" et certains agents la jugeaient frustrante et contre-productive par rapport à leur formation professionnelle. Selon Robert Lee Maril, beaucoup parmi les meilleurs agents quittèrent la police des frontières à la suite de la mise en place de cette stratégie ${ }^{(13)}$. Les militants des droits de l'homme accusèrent la police des frontières d'être responsable de ces décès ${ }^{(14)}$,à quoi les autorités répliquèrent que c'étaient les migrants et leurs passeurs qui prenaient la décision de franchir des zones dangereuses. En outre, elles insistaient sur les programmes mis en ceuvre pour sauver les migrants perdus dans le désert et pour avertir les migrants clandestins potentiels des dangers d'un franchissement illégal ${ }^{(15)}$. Le Department of Homeland Security (Département de la Sécurité intérieure), qui abrite le contrôle de l'immigration depuis 2003, percevait pour sa part ce dispositif comme un outil de prévention des blessures et des décès, tout clandestin appréhendé étant un migrant de moins qui risquait de s'aventurer dans des zones dangereuses ${ }^{(16)}$.

\section{L'impact de l'organisation de la police des frontières}

La surprise exprimée par les responsables de l'INS devant l'augmentation des décès de migrants après la mise en ceuvre de la stratégie de dissuasion soulève des questions sur la transmission des informations au sein même de la bureaucratie. Selon Sjoberg et al., les membres de la bureaucratie peuvent retenir des informations pour renforcer leur pouvoir ou éviter des blâmes de leurs supérieurs ${ }^{(17)}$. Mais, comme le suggère l'application de la dissuasion, les différences culturelles entre unités bureaucratiques peuvent aussi stopper le flux d'information quand des sous-agences ajoutent à leurs traditions locales un savoir spécialisé sans le transmettre ou en le transmettant trop lentement à l'organisation hôte. Étant donné la familiarité de la police des frontières avec la zone frontalière, les dirigeants de l'INS auraient dû anticiper l'augmentation des décès de migrants associée à la stratégie de la dissuasion. Or, il a fallu plusieurs années de rapports sociologiques sur ces décès et leurs liens avec l'application du programme pour que l'INS se décide à lancer une initiative de sécurisation des migrants à la frontière en juin $1998^{(18)}$. Les agents de la police des frontières avaient une connaissance intime du terrain où se déplacent les immigrants clandestins et étaient au courant des décès sur les routes de migration. Mais plusieurs raisons tenant à sa culture propre ont pu empêcher la police des frontières de transmettre ces informations assez tôt pour attirer l'attention de la tête de l'INS. 
Lune de celles-ci est que les agents ne jugeaient sans doute pas ces morts assez importantes pour transmettre rapidement l'information. Trouver des restes humains sur les passages de frontière, surtout les plus accidentés, est pour eux chose courante ${ }^{(19)}$. En outre, le nombre initial de décès dans les premières années suivant le lancement officiel de la stratégie de la dissuasion fut environ $30 \%$ inférieur au pic de plus 350 décès atteint en $1988^{(20)}$. Il fallut attendre plusieurs années pour que les rapports fassent état d'une augmentation spectaculaire des décès dans un secteur donné(Tucson). Une autre raison pour que ces décès n'aient pas alarmé les autorités, c'est que la police des frontières opère par secteurs ; or dans tous les secteurs le chiffre des décès resta d'abord relativement bas, et donc à un niveau "normal". En outre, les décès de migrants dans ces zones sont en général découverts individuellement, et peuvent sembler davantage la conséquence d'un comportement individuel que d'une politique.

Enfin, selon Robert Lee Maril, qui a passé deux ans à interroger les agents de la police des frontières, celle-ci est une "institution de décision du haut vers le bas ${ }^{(21)}$,, où les directeurs ont coutume d'ignorer les suggestions des agents de terrain. La direction peut qualifier les agents qui persistent à signaler des problèmes de "fauteurs de trouble" et les punir en les ridiculisant, en les harcelant, ou en leur refusant des promotions. Ces agents sont donc peu motivés à signaler un accroissement du nombre de décès dans un secteur comme l'Arizona à la suite du changement de direction des flux lié à la stratégie de dissuasion.

\section{La méconnaissance de la logique des migrants}

Les concepteurs de la stratégie de la dissuasion supposaient que la difficulté du terrain dissuaderait l'immigration clandestine dans les régions frontalières non sécurisées ${ }^{(22)}$. Le fait que des milliers de migrants clandestins aient décidé de franchir ces terrains dangereux et qu'un grand nombre aient ainsi trouvé la mort a démontré que la bureaucratie de la police des frontières manquait d'un "savoir moral" suffisant sur sa population cible. On peut définir le savoir moral comme le niveau d'information et de compréhension que doit avoir une organisation pour éviter de nuire à sa clientèle dans le cadre de ses activités.

La discussion de Anthony Giddens sur le "savoir mutuel" fournit un point de départ pour conceptualiser ce savoir dans les corps institutionnels ${ }^{(23)}$. Le savoir mutuel est plus qu'une information sur les pratiques d'un groupe ; il suppose également d'avoir une idée des informations que possède ce groupe et de l'ensemble des croyances ("théories en usage") qui guide ses membres dans leur vie quotidienne. Anthony 
Giddens semble placer le savoir mutuel plus haut que le savoir ordinaire, puisque ce dernier n'exige pas de profondeur conceptuelle au-delà de la connaissance commune dans un domaine donné, ni la conscience du caractère commun de ce savoir.

Les tentatives de franchissement de zones frontalières dangereuses par les migrants après l'application de la nouvelle stratégie ont démontré que la bureaucratie n'avait pas un savoir moral adéquat pour une mise en ceuvre humanitaire de sa stratégie de la dissuasion. Selon le témoignage devant le Congrès d'un représentant de la police des frontières, la stratégie de la dissuasion "a totalement échoué à réduire les flux d'immigration clandestine, parce que ses concepteurs ont gravement sous-estimé le degré de désespoir des pauvres dans les pays en développement ${ }^{24) " .}$

On ne peut pas supposer que les migrants internationaux connaissaient la difficulté à traverser ces terrains, beaucoup ignorant la topographie et le climat de la frontière. En outre, les concepteurs de la stratégie de la dissuasion n'avaient pas un niveau compétent de savoir mutuel sur la logique motivant la migration clandestine. La stratégie de la dissuasion se fonde sur une logique utilitaire supposant que les individus feront des choix rationnels en tentant de maximiser les avantages tout en minimisant les risques. Mais deux conditions rendent le modèle utilitaire inapplicable à la plupart des immigrants clandestins. D'abord, leur motivation première est souvent une unité familiale plutôt qu'un individu, et de nombreux migrants sont prêts à prendre des risques exceptionnels pour leur famille. Ensuite, même quand ils pèsent les avantages et les inconvénients, ces migrants opèrent dans une matrice de choix limités. Leurs ressources et leurs opportunités étant très faibles, ils tendront à prendre plus de risques que des gens mieux dotés. Enfin, la situation des plus pauvres de ces migrants est d'autant plus contrainte que, pour eux, faire demi-tour n'est pas une option.

Quant au souci des droits de l'homme, il est probable que la bureaucratie obère le savoir moral par le poids de son engagement éthique vis-à-vis de l'intégrité de l'Étatnation. Cela ne veut pas forcément dire que l'on ne se soucie plus des droits de l'homme, mais que la mission prime sur d'autres priorités. Dans les activités actuelles de contrôle, les autorités désignent souvent les migrants sous les qualificatifs d"étrangers clandestins" et "wetbacks ${ }^{(25) ", ~ q u i ~ d e ́ s h u m a n i s e n t ~ l a ~ p o p u l a t i o n ~ c i b l e, ~ l a ~}$ faisant apparaître indigne pour qu'on se soucie de ses droits ${ }^{(26)}$.

\section{Une vision négative de la migration}

La stratégie de la dissuasion n'a pas été appliquée dans un cadre neutre mais, au contraire, dans une atmosphère de sentiments nationaux d'hostilité croissante visà-vis des clandestins ${ }^{(27)}$. Cela tient en partie à un processus de construction sociale 
dépeignant la frontière comme une région en crise. Les autorités gouvernementales et les leaders politiques se sont déplacés à la frontière du Mexique dans les années quatre-vingt-dix pour accuser les clandestins de susciter des problèmes sociaux et de constituer un risque pour la sécurité nationale. La construction sociale de la frontière en tant que zone de non-droit diabolisait les clandestins perçus comme la principale menace ${ }^{(28)}$. En tournant une vidéo sur les relations publiques intitulée Border Under Siege, ("La frontière assiégée"), la bureaucratie de la police des frontières a joué elle-même un rôle direct dans l'image d'une frontière "envahie" par "des étrangers"(29).

Dans ce contexte, le contrôle devenait plus qu'un devoir : il devenait une activité morale que la bureaucratie associait de surcroît au développement de la communauté. En tant qu'outil moral, le contrôle ne pouvait être mis en cause, malgré une augmentation des décès de migrants.

Pourtant, de nouvelles recherches sont nécessaires pour déterminer dans quelle mesure les agents de base de la police des frontières se sont associés à cette vision des clandestins comme des envahisseurs représentant une menace. Les anciens agents de patrouille interrogés pour cet article indiquaient qu'ils avaient parfois des sentiments humains pour les migrants quand ils travaillaient pour la patrouille, mais qu'ils se sentaient tenus de suivre toutes les procédures d'application.

À titre de pratiques d'engagement dans la communauté, les responsables mêlaient délibérément leur identité bureaucratique aux communautés locales par le biais d'activités civiques - notamment une participation aux forums et aux cérémonies locales et la promotion des étudiants dans les écoles publiques. Au XXI ${ }^{\mathrm{e}}$ siècle, la bureaucratie du contrôle des frontières a ajouté à ses objectifs déclarés le renforcement de la qualité de vie dans les régions frontalières. Avec la frontière "sous contrôle opérationnel", affirmait-elle, la vitalité économique allait s'accroître dans les régions frontalières alors que la délinquance et les coûts des services sociaux baisseraient ${ }^{(30)}$. Par cette participation civique, la bureaucratie se présentait comme une composante éthique de la communauté.

\section{Causalité et responsabilité}

Les avocats des droits de l'homme et d'autres critiques ont attribué à la bureaucratie de la frontière la responsabilité des centaines de morts annuelles de clandestins ${ }^{(31)}$. Mais il existe un large spectre de causalité et de responsabilité des bureaucraties appliquant des politiques sociales nuisibles. Les relations causales varient en type et en degré ${ }^{(32)}$. Certaines conditions sociales entretiennent un rapport mécanique, de 
sorte que la condition B ne peut survenir que si la condition A est d'abord remplie. Cependant, les morts de migrants dans la région frontalière n'ont pas un rapport rigide, mécanique, avec la stratégie de la dissuasion, et ceci pour deux raisons. L'une est que les décès de migrants ont été enregistrés au moins une décennie avant l'apparition de cette stratégie (bien qu'avec un moindre pourcentage de morts dans le désert), et l'autre est que seul un faible pourcentage de migrants meurt après avoir tenté de franchir clandestinement la frontière.

L'association statistique caractérise un second type de causalité. Si l'on peut trouver des corrélations statistiques entre les

En tant qu'outil moral,
le contrôle ne pouvait
être mis en cause,
malgré une augmentation
des décès de migrants.
morts de migrants et des éléments de la stratégie de la dissuasion ${ }^{(33)}$, le fait qu'il y ait eu des décès de migrants en l'absence de cette stratégie et que ces morts aient fluctué de façon significative sans fluctuations similaires dans le renforcement du contrôle, indique que cette causalité n'est pas un caractère premier du rapport entre les morts de migrants et la stratégie de la dissuasion.

Un troisième type de causalité peut être caractérisé comme une causalité interactionnelle, dans laquelle les conditions et les acteurs s'influencent mutuellement. Dans la région frontalière, la migration clandestine a engendré la planification et la mise en ceuvre de la stratégie de la dissuasion, laquelle à son tour a engendré la réorientation du flux de clandestins. La causalité interactionnelle présente deux avantages analytiques pour comprendre la causalité des morts de migrants.

Le premier est qu'il n'y a pas d'influence stricte, unidirectionnelle où les acteurs auraient un rôle exclusivement dépendant ou indépendant. Le second avantage est que, les agents de la police des frontières et les migrants clandestins influant sur cette interaction, ils ont implicitement les uns et les autres la propriété de réflexivité.

Par le biais de cette réflexivité, les migrants peuvent tenter d'échapper à l'influence de la stratégie de la dissuasion, et apparemment beaucoup y ont réussi, si l'on en juge par le faible taux de décès rapporté pour le taux élevé de passages clandestins ${ }^{(34)}$.

La causalité interactionnelle ne disculpe pas la bureaucratie de rediriger les migrants vers des environnements dangereux. La stratégie de la dissuasion a réduit les choix des lieux et des moyens de passage non autorisé de la frontière, ce qui, comme on pouvait le prévoir, a accru les risques pour ceux qui persistent dans leur projet de traversée, les autres points de passage étant désormais inaccessibles. Vu les forts volumes de migration clandestine, il ne pouvait qu'y avoir des décès. 


\section{Une dynamique de renforcement des frontières}

Les politiques frontalières poursuivent l'un des objectifs les plus importants d'engagement éthique dans un système social : préserver l'intégrité spatiale de l'Étatnation. Elles affrontent de grands défis, le nombre actuel de migrants internationaux se montant selon l'IOM à une estimation de 214 millions dans le monde. Comme l'avait prévu Émile Durkheim ${ }^{(35)}$, de nombreux États-nations ont intégré des protections humanitaires à leurs législations, mais ces protections concernent souvent au premier chef les citoyens plutôt que les non-citoyens, et surtout pas les immigrants clandestins. Aux États-Unis, après l'échec en 2007 d'une proposition de loi qui aurait légalisé la présence d'une partie des 11 millions de clandestins présents dans le pays, l'Etat a insisté sur le contrôle de l'immigration, devenu en outre une mesure de sécurité nationale contre le terrorisme. Cette nouvelle politique consiste essentiellement en décisions bureaucratiques autorisant des contrôles plus nombreux à l'intérieur du pays, en un accroissement de la capacité de détention et de déportation, et un renforcement spectaculaire de la surveillance et de la clôture de la frontière ÉtatsUnis/Mexique. Pourtant, les critères des droits de l'homme s'appliquent tout à fait au traitement des migrants, puisque les États-Unis ont signé différents accords, dont la Déclaration universelle des droits de l'homme. Le plan stratégique visant à rediriger les migrants vers des environnements dangereux est incompatible avec trois articles de celle-ci, que les États-Unis ont pourtant ratifiés : "Tout individu a droit à la vie, à la liberté et la sûreté de sa personne" (art. 3), "Nul ne sera soumis à la torture, ni à des peines ou traitements cruels, inhumains ou dégradants" (art. 5), et "Chacun a le droit à la reconnaissance en tous lieux de sa personnalité juridique" (art. 6). Les clandestins poussés vers des terrains dangereux sont placés de facto dans des situations où ils connaissent des conditions inhumaines et dégradantes, et enfin la mort pour certains. En outre, les migrants qui entrent dans le pays sans autorisation doivent encore se voir garantir un statut de personne juridique. Cela inclut le droit à être entendu, le droit de présenter et réfuter des preuves, et le droit à un conseil juridique gratuit.

\section{Préserver |'ordre social mondial}

Selon Sjoberg et al., "la bureaucratie est l'outil majeur pour maintenir les inégalités dans les sociétés modernes ${ }^{(36) "}$. Mais on peut dire la même chose de la bureaucratie dans la perspective plus vaste de l'ordre social mondial. Dans leur engagement éthique à protéger l'intégrité spatiale de leurs États-nations, les bureaucraties de protection des frontières agissent pour préserver des divisions sociales et spatiales au sein de systèmes sociaux interrégionaux et mondiaux - et parfois suivant des solutions qui mettent en danger la vie des migrants.

\section{Traduit de l'espagnol par Françoise Bouillot}


* Je remercie Charles Munnell, James Pinkerton, Monalisa Chandra et Lucia Galvan pour leur aide.

1. Gideon Sjoberg, Ted R. Vaughan et Norma Williams, "Bureaucracy as a moral issue", in The Journal of Applied Behavioral Science, vol. 20, $\mathrm{n}^{\circ}$ 4, 1984, pp. 441-453.

2. Ted R. Vaughan, et Gideon Sjoberg, "The individual and bureaucracy: an alternative meadian interpretation", in The Journal of Applied Behavioral Science, $\mathrm{n}^{\circ}$ 20,1984, pp. 57-69.

3. Government Accountability Office, Illegal Immigration, Washington, D.C., décembre 1997.

4. Government Accountability Office (GAO), Border Control, Washington, D.C., décembre 1994.

5. Voir U.S. Immigration and Naturalization Service, 2001 Yearbook of Immigration Statistics, Washington, D.C., 2002, tableau 60 ; voir Karl Eschbach, Jacqueline Hagan et Nestor Rodriguez, "Deaths during undocumented migration", in In Defense of the Alien, ${ }^{\circ}$ 26, 2003, pp. 37-52, figure 1.

6. Karl Eschbach et al., "Deaths during undocumented migration", op. cit., figure 3.

7. Karl Eschbach, Jacqueline Hagan, Nestor Rodriguez, Ruben Hernandez Leon et Stanley Bailey, "Death at the border", in International Migration, $\mathrm{n}^{\circ}$ 33, 1999, pp. 430-454.

8. Government Accountability Office (GAO), Illegal Immigration, Washington, D.C., août 2006.

9. Karl Eschbach et al., "Deaths during undocumented migration", op. cit.

10. Government Accountability Office (GAO), Illegal Immigration, Washington, D.C., août 2006.

11. Ibid.

12. Ibid.

13. Robert Lee Maril, The Fence, Lubbock, Texas Tech University Press, 2011.

14. Claudia E. Smith, "Operation Gatekeeper and Migrant Deaths", in California Association of Human Relations Organizations Newsletter, 1998 ; Joseph Nevins, Operation Gatekeeper, New York, Routledge, 2002.

15. Government Accountability Office (GAO), Illegal Immigration, Washington, D.C., août 2006.

16. Ibid.

17. Gideon Sjoberg, Ted R. Vaughan et Norma Williams, "Bureaucracy as a moral issue", op. cit.

18. Government Accountability Office (GAO), Illegal Immigration, Washington, D.C., mai 1999.

19. John Annerino, Dead in their Tracks, New York, Four Walls Eight Windows, 1999.

20. Karl Eschbach et al., "Deaths during undocumented migration", op. cit.

21. Robert Lee Maril, The Fence, op.cit., p. 110.

22. Government Accountability Office (GAO), Illegal Immigration, Washington, D.C., 1994.

23. Anthony Giddens, The Constitution of Society, Berkeley, University of California Press, 1984.

24. U.S. Congress, Senate, "Subcommittee on Immigration, Border Security and Citizenship of the Committee on the Judiciary", 109 Congrès, Hearings on Federal Strategies to End Border Violence, $2^{\mathrm{c}}$ session, 2006.

25. "Dos mouillés", qui ont traversé le fleuve à la nage (N. du T.).

26. William Langewiesche, Cutting for Sign, New York, Pantheon Books, 1993.

27. Juan F. Perea (dir.), Immigrants Outs, New York, New York University Press, 1997.

28. Nestor Rodriguez, "The Social Construction of the U.S.-Mexico Border", in Juan F. Perea (dir), Immigrants Out!, New York, New York University Press, 1997 pp. 223-243.

29. Douglas S. Massey, Jorge Durand et Nolan J. Malone, Beyond Smoke and Mirrors, New York, Russell Sage Foundation, 2002.

30. U.S. Customs and Border Protection, National Border Patrol Strategy, Washington, D.C., 2004, p. 11.

31. Wayne A. Cornelius, "Death at the border", in Population and Development Review, n' 27, 2001, pp. 661-685 ; Claudia E. Smith, "Operation Gatekeeper and Migrant Deaths", op. cit.; Joseph Nevins, Operation Gatekeeper, op. cit.

32. Mario Bunge, Causality and Modern Science, New York, Dover Publications, 1979.

33. Raquel Rubio-Goldsmith, M. Melissa McCormick, Daniel Martinez et Inez Magdalena Duarte,

"The 'Funnel Effect' \& recovered bodies of unauthorized migrants processed by the pima county office of the medical examiner, 1990-2005", University of Arizona, Mexican American Studies and Research Center, 2006.

34. Douglas S. Massey, Jorge Durand et Nolan J. Malone, Beyond Smoke and Mirrors, op. cit.

35. Émile Durkheim, L'Éducation morale, Paris, PUF, 1963.

36. Gideon Sjoberg, Ted R. Vaughan et Norma Williams, "Bureaucracy as a moral issue", op. cit., p. 446. 